\title{
Effect of Stochastic Dead Space on Noise in Avalanche Photodiodes
}

\author{
Oh-Hyun Kwon, Majeed M. Hayat, Senior Member, IEEE, Joe C. Campbell, Fellow, IEEE, \\ Bahaa E. A. Saleh, Fellow, IEEE, and Malvin Carl Teich, Fellow, IEEE
}

\begin{abstract}
A stochastic dead-space model for impact ionization is developed and used to study the effect of the soft nature of the ionization capability of carriers on the excess noise factor of avalanche photodiodes. The proposed model is based on the rationale that the gradual, or soft, transition in the probability density function (PDF) for the distance from birth to impact ionization can be viewed as that resulting from uncertainty in the dead space itself. The resulting soft PDF, which is parameterized by a tunable softness parameter, is used to establish the limitations of the existing hard-threshold ionization models in ultrathin multiplication layers. Calculations show that for a fixed operational gain and fixed average dead space, the excess noise factor tends to increase as a result of the softness in the PDF in very thin multiplication layers (viz, $<70 \mathrm{~nm}$ ), or equivalently, under high applied electric fields (viz., $>800 \mathrm{kV} / \mathrm{cm}$ ). A method is proposed for extracting the softness parameter from noise versus multiplication measurements.
\end{abstract}

Index Terms-Avalanche photodiodes (APDs), excess noise factor, GaAs, impact ionization, ionization coefficient, ionization threshold energy, stochastic dead space (SDS).

\section{INTRODUCTION}

I T HAS become evident in recent years that the excess noise factor in avalanche photodiodes (APDs) with thin multiplication layers (viz., $<200 \mathrm{~nm}$ ) is significantly lower than that originally anticipated by the McIntyre multiplication theory [1]. This is now known to be a result of the dead space, which is the minimum distance that a carrier must travel in order to acquire sufficient energy enabling it to impact ionize [2]-[7]. Notably, the effect of dead space on the carrier multiplication process has been shown to become more pronounced as the thickness of the multiplication layer is reduced [2]-[4]. As the device becomes thinner (while holding the mean gain fixed), dead space begins to occupy a larger fraction of the multiplication region and its effect on the excess noise factor becomes substantial.

Since the time when Spinelli and Lacaita [8] pointed out the significance of the dead-space effect in thin APDs, there have

Manuscript received October 24, 2003; revised January 6, 2004. This work was supported by the National Science Foundation under Award ECS-0196569. The review of this paper was arranged by Editor L. Lunardi.

O.-H. Kwon and M. M. Hayat are with the Department of Electrical and Computer Engineering, University of New Mexico, Albuquerque, NM 87131-1356 USA (e-mail: hayat@eece.unm.edu).

J. C. Campbell is with the Department of Electrical and Computer Engineering, University of Texas at Austin, Austin, TX 78712-1100 USA (e-mail jcc@mail.utexas.edu).

B. E. A. Saleh and M. C. Teich are with the Department of Electrical and Computer Engineering, Boston University, Boston, MA 02215-2421 USA (e-mail: besaleh, teich@bu.edu).

Digital Object Identifier 10.1109/TED.2004.825798 been many APDs developed whose noise characteristics could not be explained without considering the dead-space effect. Hu et al. [9] experimentally demonstrated the noise reduction in a thin multiplication region GaAs APD and attributed it to the "size effect" (i.e., dead-space effect). Later, Li et al. [2] demonstrated the significance of the dead space in thin multiplication layers (GaAa) and Ong et al. [10] used the dead-space analytical model to account for the noise reduction. Their results were also tested using Monte Carlo simulation, as reported in [3]. Subsequently, Yuan et al. [11] showed the effect using McIntyre's history-dependent theory [6]. At the same time, Saleh et al. [4], [5] explained the noise reduction of GaAs, AlGaAs, InAlAs, and InP APDs using the recurrence theory developed by Hayat et al. [12], [13]. Most recently, Beck et al. [14] and Ma et al. [15] demonstrated the noise-free $\mathrm{HgCdTe}$ APD and reasoned the noise characteristics with the dead-space effect [6]. Thus, the dead-space model is not only useful in near-infrared devices, but it also extends to midinfrared applications. In addition to noise analysis, the dead-space effect also applies to the speed analysis as shown by $\mathrm{Ng}$ et al. [16], Hambleton et al. [17], and Hayat et al. [18]. In addition, the dead-space model has evolved to demonstrate even lower than expected noise due to the so-called initial-energy effect and the heterojunction effect in bandgap engineered heterostructure APDs [19]-[22]. The dead-space model can be used to design and optimize such heterostructure APDs. Finally, recent studies have also shown that the dead space impacts the breakdown probability characteristics as a function of the reverse-bias voltage [23], [24].

A convenient and simple way to model the dead space is to assume that the density of impact ionization (i.e., the ionization coefficient) is zero before the dead space, after which it abruptly assumes a constant rate (namely, the ionization coefficient of enabled carriers that have reached an equilibrium energy distribution). With this assumption, Okuto and Crowell [25] developed a model to calculate the mean multiplication gain. Later, Hayat et al. [12], [13], [26] developed a recursive technique that facilitated the calculation of the excess noise factor and characterized the probability distribution of the gain. We refer to this model as the hard-threshold dead-space multiplication theory (HDSMT). In fact, the HDSMT model is the spatial analog of the fixed nonparalyzable dead-time-modified Poisson process [27]-[29]; the underlying Poisson character of this process is responsible for the exponential behavior of the interevent intervals for distances greater than the dead space. Using the HDSMT and its more recent variants, good agreement with experimental measurements has been observed for multiplication-region thicknesses down to 
$100 \mathrm{~nm}$ [4], [5], [21]. From a physical viewpoint, however, one would expect the ionization density to gradually increase from zero to its constant steady-state value. Indeed, Monte Carlo simulation studies [3], [30]-[32] have shown a smooth rise in the ionization probability density (the ionization rate) from zero to a steady-state value, which demonstrates the soft nature of the ionization ability of carriers, as discussed in more detail below.

In some physical processes, including impact ionization, the dead space is stochastic, rather than fixed (which gives rise to the variable nonparalyzable dead-time modified Poisson process [33]). The principal cause of this randomness of the dead space in impact ionization is twofold: 1) There is uncertainty in the actual energy that a carrier must accumulate in order to reach the ionization threshold. This is due to the fact that there is uncertainty in a carriers (both injected and offspring) energy prior to ionization. For example, unlike the implicitly-adopted assumption in the HDSMT, a parent carrier may not lose all of its accumulated energy upon impact ionizing but may retain some residual energy. At the same time, an offspring carrier may be generated with nonzero energy. Moreover, Sano et al. [34] pointed out that carriers are not necessarily travelling parallel to the field direction and thus, their momenta are random. Therefore, the ionization threshold energy depends on the wave vectors of initiating carriers, and this brings about uncertainty in the ionization threshold energy itself. 2) The occurrence of phonon scattering, as the carrier travels through the high-field region, also impacts the total energy that it must accumulate as it can release or absorb energy by phonon scattering. In fact, using Monte Carlo simulation Plimmer et al. [35] have observed that the softness in the ionization ability of carriers caused by phonon scattering is more significant than that caused by uncertainty in the initial energy of carriers.

In other circumstances, carriers can be gradually, rather than abruptly, enabled to impact ionize, resulting in relative-dead space model (also called a sick-space model or a soft-threshold ionization rate model), which is the spatial analog of the sicktime-modified Poisson process [36]. In particular, once a carrier has reached the ionization threshold energy, it may ionize at a rate that depends on the energy it has in excess of the ionization threshold energy. Naturally, this effect brings about softness in the ionization rate beyond the ionization threshold energy. Despite their distinct origins, both the stochastic dead-space (SDS) effect and the relative dead-space effect result in a softness in the ionization capability of carriers.

Monte Carlo studies suggest that the effect of the softness in the ionization capability becomes significant in the analysis of very thin devices (below $100 \mathrm{~nm}$ ) and high electric fields. For example, Ong et al. [10] showed that the noise of a 50-nm device is not accurately predicted by the HDSMT. Recently, an analytical study was undertaken by Tan et al. [37] to capture the threshold softness. They proposed a soft-threshold ionization (or a relative dead-space approach) model, which permitted the gradual increase of the ionization probability from zero to the steady-state value. Their method involved employing a shape function, taken as the indefinite integral of a Gaussian function with a width parameter $s$, which would control the smoothness of the transition of the ionization probability (a small $s$ value would give a sharp transition while a large $s$ value would yield a more gradual transition). Tans model was applied to a Si APD and the $s$ parameter was fitted to measured excess noise data (the HDSMT model, in this case, would predict higher noise than measurement). Despite the success of Tans work in capturing the softness of the ionization capability, there are some key questions that remain unanswered. For example, as $s$ increases, so does the effective dead space; thus, it is not clear whether the reduced noise suggested in Tans work is mainly a result of the softness of the ionization ability, the elongated effective dead space, or both. Intuitively, if we keep the effective dead space constant while softening the ionization ability (without changing the mean multiplication), we would expect the noise to increase, as the softness will add to the overall uncertainty of the multiplication process.

In this paper, we make use of the SDS approach to capture the softness in the ionization capability of carriers and study its effect on the excess noise factor. In particular, our intent is to perform a systematic study to show at which thicknesses the accuracy of the HDSMT model becomes questionable. We perform this by systematically introducing uncertainty in the dead space, which successfully resulted in various degrees of softness in the ionization probability. The approach is based on randomizing the dead space in the HDSMT model, which results in a soft probability density function (PDF) for the distance from the carriers birth to impact ionization. This soft PDF contains a tunable parameter governing the variability range of the dead space, which in turn, controls the degree of softness in the ionization ability of carriers. In comparison to the relative dead-space (sick-space) model, this approach obviates the need for postulating an ad hoc recovery function for the sick space while providing an adequate representation of the softness in the ionization capability of carriers.

\section{SDS MODEL}

As stated earlier, we will regard the dead space as a random variable, which is used, in turn, in a randomized version of the HDSMT model to obtain a PDF of the ionization distance. Consider a multiplication region extending from $x=0$ to $x=w$, and let $D_{e}(x)$ denote the random dead space for an electron born at location $x$. Now suppose that $\delta$ is a particular realization of the random dead space $D_{e}(x)$. Then, conditional on $D_{e}(x)=\delta$ and according to the HDSMT, the PDF of the location $\xi$ (measured relative to $x=0$ ) at which the electron impact ionizes has the shifted-exponential form given by [12], [20]

$h_{e}\left(\xi \mid x, D_{e}(x)=\delta\right)= \begin{cases}0, & \xi<x+\delta \\ \alpha(\xi) \exp \left(-\int_{x+\delta}^{\xi} \alpha(u) d u\right), & \xi \geq x+\delta\end{cases}$

where it is understood that $\delta$ is a particular realization of the random dead space. Here, $\alpha(x)$ is the nonlocalized ionization coefficient for the electron, that is, the ionization coefficient for electrons that have already travelled the dead space. This ionization coefficient is assumed to be position dependent through its dependence on the nonuniform electric field. 
Next, we will ensemble average the above conditional PDF, given by (1), over all possible realizations of the random dead space and obtain the unconditional PDF (with the dead-space averaged) given by

$$
\begin{aligned}
h_{e}(\xi \mid x) & =\mathrm{E}\left[h\left(\xi \mid x, D_{e}(x)\right)\right] \\
& =\int_{0}^{\xi-x}\left(\alpha(\xi) e^{-\int_{x+\delta}^{\xi} \alpha(u) d u}\right) f_{D_{e}(x)}(\delta) d \delta
\end{aligned}
$$

where the expectation operator " $E$ " is taken with respect to the PDF of the random dead space $D_{e}(x)$, which is denoted in (2) by $f_{D_{e}(x)}(\cdot)$. In the case of a spatially uniform nonlocalized ionization coefficient $\alpha$, (2) can be determined with the knowledge of the PDF of the dead space. For simplicity, if we assume that $D_{e}(x)$ is uniformly distributed in the interval $\left[d_{\min }, d_{\max }\right]$, then calculations yield

$$
h_{e}(\xi \mid x)=\left\{\begin{array}{lr}
0, & \xi<x+d_{\min } \\
\frac{1}{\Delta d}\left[1-e^{-\alpha\left(\xi-x-d_{\min }\right)}\right], & x+d_{\min } \leq \xi \\
\frac{1}{\Delta d} e^{-\alpha(\xi-x)}\left[e^{\alpha d_{\max }}-e^{\alpha d_{\min }}\right], & \xi>x+d_{\max } \\
\text { max }
\end{array}\right.
$$

where $\Delta d=d_{\max }-d_{\min }$.

Let us now examine the key properties of the above PDF, as shown in Fig. 1. First, the PDF is zero when $\xi<x+d_{\min }$. Second, the point $\xi=x+d_{\min }$ is where the PDF starts to rise and $\xi=x+d_{\max }$ is the point where the PDF reaches its peak value; and third, for $\xi>x+d_{\max }$, the PDF decays exponentially at a rate $\alpha$. Thus, we have arrived at a PDF that exhibits a soft ionization capability, where the peak value is gradually attained over a distance $\Delta d$ beyond a minimal-dead-space point where the PDF begins to ascend from 0. Clearly, when $\Delta d=0$, in which case the dead space is deterministic, the PDF in (3) collapses to the HDSMT PDF, as given by (1). Note that the dead-space uncertainty $\Delta d$ can be interpreted as uncertainty in the energy, $\Delta E$, needed to be acquired from the field in order to achieve the ionization threshold energy: $\Delta E=q \mathcal{E} \Delta d$, where $q$ is the electronic charge and $\mathcal{E}$ is the applied electric field. We call the PDF of the form shown in Fig. 1 a soft $\mathrm{PDF}$, and parameter $\Delta E$ is termed the softness parameter, as it controls the softness in the transition from zero to the peak value of the soft PDF. It is important to point out that the choice of a uniform distribution for the dead space ultimately affects the shape of the soft PDF, and in particular, it is responsible for its sharp peak at the designated ionization distance. Conceivably, we would expect that if a more realistic distribution is used, the sharpness of the peak will be reduced yielding a theoretical PDF which better resembles that obtained from Monte Carlo simulations [10], [35]. The choice of a uniform distribution, in this case, resulted from our insufficient analytical knowledge of the true distribution of the dead space. This distribution, nonetheless, captures the dead-space randomness using a minimum number of unknown parameters (namely, $\Delta E$ ).

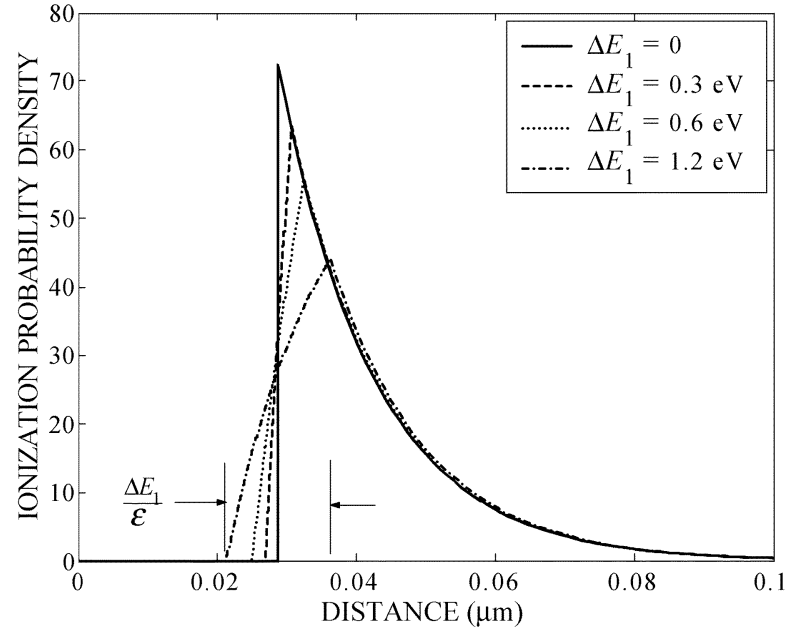

Fig. 1. Soft probability density function of the ionization distance. The average dead space is assumed fixed and the softness parameter $\Delta E_{1}$ is varied. The arrows indicate the degree of softness introduced in the PDF as a result of $\Delta E_{1}=1.2 \mathrm{eV}$.

In the next section we will use the average distance between ionization events, $\langle l\rangle$, in the calculation of the ionization rates to be used in conjunction with the soft PDF (3). The average interionization distance is

$$
\langle l\rangle=\int_{0}^{\infty}(\xi-x) h(\xi \mid x) d \xi
$$

and in the case of a uniform electric field, $\langle l\rangle$ turns out to be

$$
\begin{aligned}
\langle l\rangle & =\alpha^{-1}+\left\langle D_{e}\right\rangle \\
& =\alpha^{-1}+\frac{\left(d_{\max }+d_{\min }\right)}{2} .
\end{aligned}
$$

With the soft PDF (3) at hand, we proceed to study the effect of the stochastic dead space on the excess noise factor in thin APDs.

\section{RESUlTS AND DisCUSSION}

The impact of the SDS model on the excess noise factor can be studied by observing the effect of varying the softness parameter in the soft PDF while fixing the average dead space. This observation aims to characterize the sensitivity of the recursive technique for computing the excess noise factor [26] to the stochastic nature of the dead space (viz., the dead-space range $\Delta d$, or equivalently $\Delta E$ ). In particular, we will establish range of the multiplication-region width over which incorporating the stochastic dead space would be important. Then, we will conform the SDS model to a physical setting where we fix the minimum dead space (corresponding to a minimal theoretical ionization threshold) and introduce an appropriate range for the dead-space uncertainty. This would provide a method for extracting the softness parameter from the APDs noise-versus-gain data in a realistic setting for which the dead space is assumed to be random in an unknown range but with a known lower bound. 


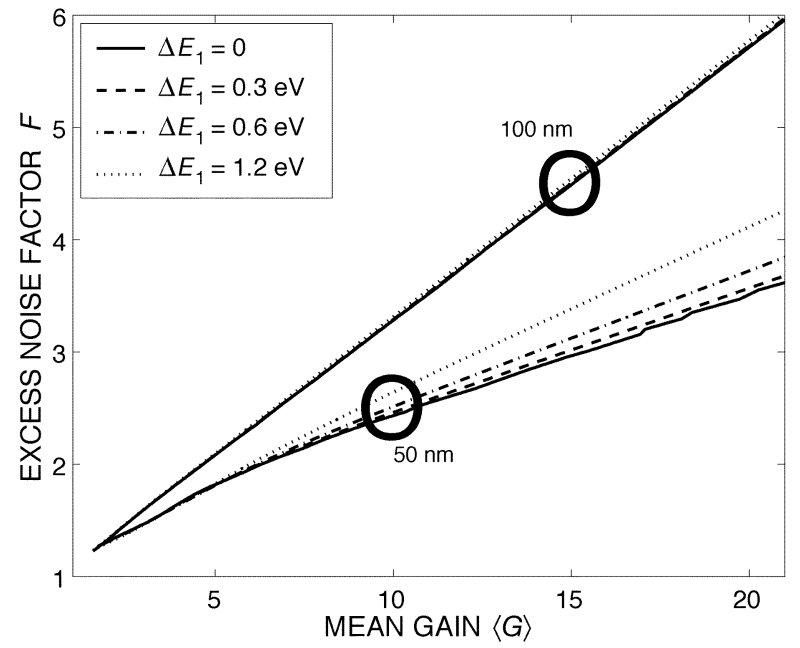

Fig. 2. Predicted excess noise factor $F$ versus the mean gain $\langle G\rangle$ for a $100-\mathrm{nm}$ GaAs APD using the SDS model. The parameter $\Delta E_{1}$ is varied while the average dead space is held fixed.

\section{A. Sensitivity of the Excess Noise Factor to Dead Space Uncertainty}

Here, we keep the average dead space in the SDS model fixed and gradually vary the uncertainty $\Delta d$ about it through varying $\Delta E$. This will restrict our attention to the effect of the dead-space uncertainty without altering the average length of the dead space. We use the average dead spaces, $\left\langle D_{e}\right\rangle=\bar{d}_{e}$ and $\left\langle D_{h}\right\rangle=\bar{d}_{h}$, which are computed from the effective ionization threshold energies of $2.3 \mathrm{eV}$ for electrons and $2.1 \mathrm{eV}$ for holes, as reported in [10]. We used the Monte Carlo simulation results reported in [10] to obtain the effective ionization coefficients $\alpha_{M C}$ and $\beta_{M C}$, for the electrons and holes, respectively. Since the average ionization length is simply the reciprocal of the ionization coefficients obtained from the Monte Carlo simulation, we set $\left\langle l_{e}\right\rangle=\alpha_{M C}^{-1}$ and $\left\langle l_{h}\right\rangle=\beta_{M C}^{-1}$ and find the nonlocalized ionization coefficients $\alpha$ and $\beta$ which are to be used in the SDS model given in (3). This technique for finding the nonlocalized coefficients was first introduced (to the best of our knowledge) by Spinelli and Lacaita [8] and yields the following simple formulas:

$$
\alpha^{-1}=\alpha_{M C}^{-1}-\bar{d}_{e}
$$

and

$$
\beta^{-1}=\beta_{M C}^{-1}-\overline{d_{h}} .
$$

The soft PDFs of the impact ionization distance computed using (3) with $x=0$ and an applied electric field of $800 \mathrm{kV} / \mathrm{cm}$ are shown in Fig. 1 for $\Delta E_{1}=0,0.3,0.6$, and $1.2 \mathrm{eV}$. (The subscript in $\Delta E_{1}$ is used to distinguish the case of variation about the mean from the case of variation $\Delta E_{2}$ beyond the minimal ionization energy, which will be considered in Section III-B.)

Estimates of the excess noise factor are shown in Fig. 2, which were generated using Hayat's recursive technique [26]. It is seen from the figure that the noise characteristics for a $100-\mathrm{nm} \mathrm{GaAs}$ APD is nearly insensitive to variation in the softness parameter $\Delta E_{1}$. The result indicates that for this multiplication-region width, the added softness does not affect the noise characteristics as long as the mean dead space is held fixed. In contrast, for a 50-nm multiplication region, the excess noise increases substantially as the softness is introduced. (In fact, this effect starts to become significant when the width begins to drop below $70 \mathrm{~nm}$ ). This is in agreement with our intuition in that the stochastic nature of the dead space brings about an additional source of randomness in the avalanche multiplication process (which tends to de-emphasize the localization brought about by the dead-space phenomenon), which, in turn, would degrade the noise characteristics. We also studied significance of the degree of softness by computing the excess noise factor as a function of the multiplication region width for two cases corresponding to the hard-threshold dead-space case (i.e., $\Delta E_{1}=0$ ), which is used as a reference, and a maximal SDS case (corresponding to $\Delta E_{1}=1.2 \mathrm{eV}$ ). The gain was held constant at 20 . We found that the stochastic dead space did not significantly alter the noise prediction of a 100-nm APD (a mere 1\% increase). In contrast, the noise estimate increased by more than $5 \%$ for a 70-nm APD and by approximately $18 \%$ for a $50-\mathrm{nm}$ APD. Indeed, this agrees with our expectation since as the thickness of the multiplication region decreases, the uncertainty in the dead space occupies a larger fraction of the multiplication region and its impact on the excess noise factor becomes more significant. The results here are also in qualitative agreement with the Monte Carlo studies reported by Ong et al. [10], which concluded that the noise of the softness-threshold model is greater than the noise of the hard-threshold model for thin devices.

\section{B. Application to Experimental Data}

Next, we employ the form of the SDS PDF (3) but set the minimum dead space $d_{\min }$ according to the minimum possible theoretical threshold energy, $E_{\mathrm{th}, \mathrm{min}}$. The minimum dead spaces, $d_{\mathrm{e}, \min }$ and $d_{\mathrm{h}, \min }$, for electrons and for holes, respectively, are obtained from the minimum ionization threshold energies, which are $1.7 \mathrm{eV}$ for electrons and $1.4 \mathrm{eV}$ for holes [38]. We then select the softness parameter $\Delta E_{2}$ that yields a good match with experimental results. Clearly, introducing variability in the dead space, beyond the minimum dead space, will elongate the average dead space. Thus, in this setting we introduce the precise dead-space variability, beyond a physically minimum dead space, that would render the correct excess-noise prediction. As before, the nonlocalized ionization coefficients, $\alpha$ and $\beta$, for the soft PDF can be found by equating the average ionization distance to the reciprocal of the effective ionization coefficients obtained by Monte Carlo simulation [10]. This yields the following formulas:

$$
\alpha^{-1}=\alpha_{M C}^{-1}-\left(d_{\mathrm{e}, \min }+\frac{\Delta d}{2}\right)
$$

and

$$
\beta^{-1}=\beta_{M C}^{-1}-\left(d_{\mathrm{h}, \min }+\frac{\Delta d}{2}\right) .
$$

Thus, for every degree of softness in the dead space, there is a corresponding nonlocalized SDS ionization coefficient. 


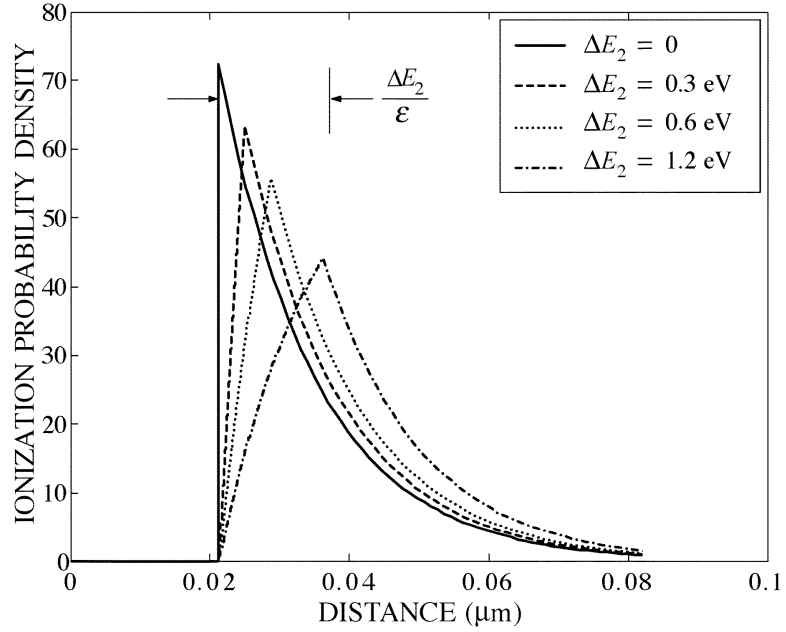

Fig. 3. Soft probability density function of the ionization distance assuming a fixed minimum dead space while the softness parameter $\Delta E_{2}$ is varied. Note that in contrast to Fig. 1, the average dead space increases as $\Delta E_{2}$ increases. The arrow indicates the degree of softness introduced in the PDF as a result of $\Delta E_{2}=1.2 \mathrm{eV}$. Note the dead space is elongated by $\Delta E_{2} / 2 q \mathcal{E}$ beyond the minimum dead space.

The PDF of the impact ionization distance for electrons is shown in Fig. 3. We emphasize that the ionization thresholds here are different from that shown earlier in Fig. 1. (Here, each ionization threshold corresponds to $E_{\mathrm{th}}=E_{\mathrm{th}, \min }+\Delta E_{2} / 2$, where the softness parameter $\Delta E_{2}$ is allowed to vary, whereas in Fig. $1, E_{\text {th }}$ is fixed at the effective ionization threshold energy provided by [5].) As $\Delta E_{2}$ increases, the location where the PDF reaches its maximum moves away from the minimal dead space, representing the increase of the softness as well as the mean dead space. These PDFs are used, in turn, to compute the excess noise factor according to the recursive technique [26]. Generally, our computations show that the excess noise factor is reduced as $\Delta E_{2}$ increases, as shown in Fig. 4. This is primarily due to the fact that when the softness is introduced, the average dead space is also extended, which tends to reduce the excess noise factor and dominate the opposite noise increase accompanying the increase in the softness parameter (the effect that was demonstrated in Section III-A). Thus, the excess noise factor is reduced overall. This observation is similar in nature to that obtained by Tan et al. in which a soft-threshold model (relative dead space model) was employed [37]. Note that $\Delta E_{2}=1.2 \mathrm{eV}$ produces an equivalent soft-threshold PDF (and thus identical noise characteristics) to that corresponding to the case $\Delta E_{1}=1.2$ (shown in Figs. 1 and 2).

The fitting of the SDS model to experimental data is considered next.

1) Extraction of the Softness Parameter: We will investigate the values of $\Delta E_{2}$ that show good agreement with the experimental noise-versus-gain measurements. Also, to have a better feel for the relative significance of the fitted $\Delta E_{2}$ and its dependence on the multiplication-region width, we introduce the field-independent, normalized dead-space variability parameter, $\rho$, defined as

$$
\rho=\frac{\Delta d}{\bar{d}}=\frac{\Delta d}{d_{\min }+\frac{1}{2} \Delta d}=\frac{\Delta E_{2}}{E_{\mathrm{th}, \min }+\frac{1}{2} \Delta E_{2}} .
$$

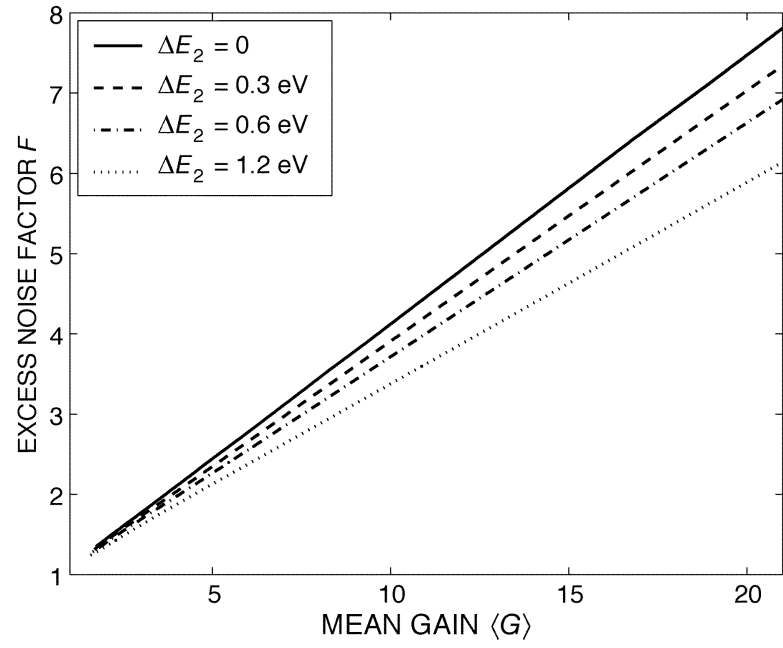

Fig. 4. Predicted excess noise factor, $F$, versus the mean gain, $\langle G\rangle$, of a $100-\mathrm{nm}$ GaAs APD. The curves are parameterized by the softness parameter $\Delta E_{2}$ but the minimum dead space is fixed at a minimum value of $E_{\mathrm{th}, \min } / 2 q \mathcal{E}$.

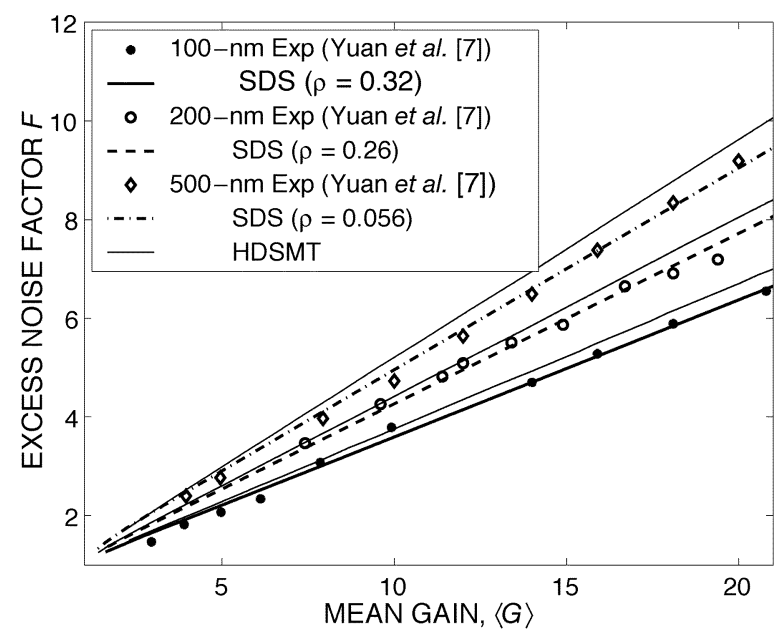

Fig. 5. Comparisons between the experimental measurements of the excess noise factor $F$ (obtained from Yuan et al. [7]) and the corresponding predictions of the SDS model. In the SDS model, the parameter $\Delta E_{2}$ (or $\rho$ ) is selected so that the computed noise-versus-gain predictions best fit the data. For comparison, the thin solid curves represent the HDSMT predictions of the excess noise factor for each device.

The results are shown in Fig. 5 for 100-, 200-, and 500-nm GaAs APDs. In these computations, the minimum ionization threshold energies of $1.7 \mathrm{eV}$ for electrons and $1.4 \mathrm{eV}$ for holes are applied according to [38]. For the 100-nm GaAs APD, the SDS model with $\Delta E_{2}=0.8 \mathrm{eV}(\rho=0.32)$ shows good agreement with experiment. For the 200-nm GaAs APD, $\Delta E_{2}=$ $0.6 \mathrm{eV}(\rho=0.26)$ is required, and for the 500-nm GaAs APD, $\Delta E_{2}=0.1 \mathrm{eV}(\rho=0.056)$. Thus, our calculations indicate that the required relative variability in the dead space, represented by the steady increase in the $\rho$ parameter as the width decreases, becomes more significant as the applied electric field increases, which is consistent with the results of Monte Carlo simulations [3], [10], [31]. Although we were able to empirically estimate 
the softness parameter from data fitting, no theoretical expression is available at the present time which would estimate its magnitude for a specific material as a function of the applied electric field.

For comparison, the thin curves in Fig. 5 represent the noise predictions using the HDSMT. It should be noted that the parameters of the HDSMT model reported in [5] (the effective ionization thresholds, in particular) are obtained by fitting the HDSMT model to noise-versus-gain data. Thus, the HDSMT effectively incorporates the dead-space softness by generating an effective average dead space (corresponding to the effective, or dead-space inclusive, ionization threshold energies). This is why the HDSMT models work reasonably well as long as the multiplication-region width falls within the range of devices whose data were used in generating the HDSMT model parameters. However, its accuracy is questionable when the multiplication-region width is further lowered primarily because it does not explicitly capture the ionization-capability softness phenomena and the fact that the thinnest APD used in its model parameter fitting had a 100-nm multiplication region [4], [5]. The SDS model, on the other hand, has a tunable parameter $\Delta E$ that allows capturing the softness in the ionization capability, thus naturally providing an improved estimate of the noise at the slight expense of model complexity.

\section{CONCLUSION}

We have examined the noise-gain characteristics of an APD in which the impact ionization is characterized by a soft PDF, with variable softness. This PDF is obtained using a model in which the dead space is taken to be random. The outcome is a gradual transition of the ionization PDF from zero to a peak value followed by an exponential tail. The tail is associated with an ionization coefficient for enabled carriers, i.e., carriers that have reached an equilibrium energy distribution in the electric field and scattering environment. In effect, therefore, this paper studies the effect of different PDF shapes on the excess noise factor versus gain characteristics of APDs. Though the shape of the PDF is significant in the calculations, the physical origin of the shape is not. The calculations which we carried out make use of a doubly stochastic approach, i.e., a fixed dead space model in which there is an underlying stochasticity of the dead space itself. The result is a soft PDF. Were the same soft PDF profile generated by different underlying physics, resulting from an alternative stochastic phenomenon, the recurrence model would nonetheless remain unchanged and identical performance would obtain.

Our calculations of the noise show that if the average dead space is held fixed, the uncertainty in the dead space does not significantly alter the excess noise predictions unless the multiplication region width is ultrathin (viz., $<70 \mathrm{~nm}$ ). Thus, for APDs with multiplication-region widths as low as $100 \mathrm{~nm}$, the commonly used hard-threshold dead space models provide adequate accuracy since incorporating the stochastic dead space does not change the predictions significantly.
In ultra-thin APDs, on the other hand, the softness of the dead space tends to increase the excess noise factor as the dead space uncertainty counteracts the orderliness that the hard-threshold dead space normally brings about. We have also developed a method for extracting the softness parameter based on noise versus gain data to illustrate the behavior of the noise characteristics as the softness is introduced. The method relies on setting the minimal dead space and selecting the dead space uncertainty range that generates the correct noise prediction. It was found that the dead-space uncertainty, normalized by the average dead space, increases as the devices become thinner, which would result in a more significant impact on the noise. That is, the model confirms that the phenomenon of the soft ionization capability becomes significant only at high fields.[15]

\section{ACKNOWLEDGMENT}

The authors wish to thank Dr. J. P. R. David and Dr. G. J. Rees at the University of Sheffield, U.K., for many valuable discussions and suggestions.

\section{REFERENCES}

[1] R. J. McIntyre, "Multiplication noise in uniform avalanche photodiodes," IEEE Trans. Electron Devices, vol. ED-13, pp. 164-168, Jan. 1966.

[2] K. F. Li, D. S. Ong, J. P. R. David, G. J. Rees, R. C. Tozer, P. N. Robson, and R. Grey, "Avalanche multiplication noise characteristics in thin GaAs $\mathrm{p}^{+}-\mathrm{i}-\mathrm{n}^{+}$diodes," IEEE Trans. Electron Devices, vol. 45, pp. 2102-2107, Oct. 1998.

[3] D. S. Ong, K. F. Li, G. J. Rees, G. M. Dunn, J. P. R. David, and P. N. Robson, "A Monte Carlo investigation of multiplication noise in thin $p^{+}-i-n^{+}$GaAs avalanche photodiodes," IEEE Trans. Electron Devices, vol. 45, pp. 1804-1810, Aug. 1998.

[4] M. A. Saleh, M. M. Hayat, B. E. A. Saleh, and M. C. Teich, "Dead-spacebased theory correctly predicts excess noise factor for thin GaAs and $\mathrm{AlGaAs}$ avalanche photodiodes," IEEE Trans. Electron Devices, vol. 47, pp. 625-633, Mar. 2000.

[5] M. A. Saleh, M. M. Hayat, P. P. Sotirelis, A. L. Holmes Jr., J. C. Campbell, B. E. A. Saleh, and M. C. Teich, "Impact-ionization and noise characteristics of thin III-V avalanche photodiodes," IEEE Trans. Electron Devices, vol. 48, pp. 2722-2731, Dec. 2001.

[6] R. J. McIntyre, "A new look at impact ionization-part I: A theory of gain, noise, breakdown probability, and frequency response," IEEE Trans. Electron Devices, vol. 46, pp. 1623-1631, Aug. 1999.

[7] P. Yuan, K. A. Anselm, C. Hu, H. Nie, C. Lenox, A. L. Holmes Jr., B. G. Streetman, J. C. Campbell, and R. J. McIntyre, "A new look at impact ionization-part II: gain and noise in short avalanche photodiodes," IEEE Trans. Electron Devices, vol. 46, pp. 1632-1639, Aug. 1999.

[8] A. Spinelli and A. L. Lacaita, "Mean gain of avalanche photodiodes in a dead space model," IEEE Trans. Electron Devices, vol. 43, pp. 23-30, Jan. 1996.

[9] C. Hu, K. A. Anselm, B. G. Streetman, and J. C. Campbell, "Noise characteristics of thin multiplication region GaAs avalanche photodiodes," Appl. Phys. Lett., vol. 69, pp. 3734-3736, Dec. 1996.

[10] D. S. Ong, K. F. Li, G. J. Rees, J. P. R. David, and P. N. Robson, "A simple model to determine multiplication and noise in avalanche photodiodes," J. Appl. Phys., vol. 83, pp. 3426-3428, Mar. 1998.

[11] P. Yuan, C. C. Hansing, K. A. Anselm, C. V. Lenox, H. Nie, A. L. Holmes Jr., B. G. Streetman, and J. C. Campbell, "Impact ionization characteristics of III-V semiconductors for a wide range of multiplication region thicknesses," IEEE J. Quantum Electron., vol. 36, pp. 198-204, Feb. 2000.

[12] M. M. Hayat, W. L. Sargeant, and B. E. A. Saleh, "Effect of dead space on gain and noise in $\mathrm{Si}$ and GaAs avalanche photodiodes," IEEE $J$. Quantum Electron., vol. 28, pp. 1360-1365, 1992. 
[13] B. E. A. Saleh, M. M. Hayat, and M. C. Teich, "Effect of dead space on the excess noise factor and time response of avalanche photodiodes," IEEE Trans. Electron Devices, vol. 37, pp. 1976-1984, Oct. 1990.

[14] J. D. Beck, C.-F. Wan, M. A. Kinch, J. E. Robinson, F. Ma, and J. C. Campbell, "The HgCdTe electron avalanche photodiode," LEOS, vol. II, pp. 849-850, 2003

[15] F. Ma, X. Li, J. C. Campbell, J. D. Beck, C.-F. Wan, and M. A. Kinch, "Monte Carlo simulations of $\mathrm{Hg}_{0.7} \mathrm{Cd}_{0.3} \mathrm{Te}$ avalanche photodiodes and resonance phenomenon in the multiplication noise," Appl. Phys. Lett. vol. 83, pp. 785-787, July 2003.

[16] J. S. Ng, C. H. Tan, B. K. Ng, P. J. Hambleton, J. P. R. David, G. J. Rees, A. H. You, and D. S. Ong, "Effect of dead space on avalanche speed," IEEE Trans. Electron Devices, vol. 49, pp. 544-549, Apr. 2002.

[17] P. J. Hambleton, S. A. Plimmer, J. P. R. David, and G. J. Rees, "Simulated current response in avalanche photodiodes," J. Appl. Phys., vol. 91, pp. 2107-2111, Feb. 2002

[18] M. M. Hayat, O. Kwon, Y. Pan, P. Sotirelis, J. C. Campbell, B. E. A. Saleh, and M. C. Teich, "Gain-bandwidth characteristics of thin avalanche photodiodes," IEEE Trans. Electron Devices, vol. 49, pp. 770-781, May 2002.

[19] P. Yuan, S. Wang, X. Sun, X. G. Zheng, A. L. Holmes Jr., and J. C. Campbell, "Avalanche photodiodes with an impact-ionization-engineered multiplication region," IEEE Photon. Technol. Lett., vol. 12, pp. 1370-1372, Oct. 2000.

[20] M. M. Hayat, O. Kwon, S. Wang, J. C. Campbell, B. E. A. Saleh, and M. C. Teich, "Boundary effects on multiplication noise in thin heterostructure avalanche photodiodes: theory and experiment," IEEE Trans. Electron Devices, vol. 49, pp. 2114-2123, Dec. 2002.

[21] O. Kwon, M. M. Hayat, S. Wang, J. C. Campbell, A. L. Holmes Jr., B. E. A. Saleh, and M. C. Teich, "Optimal excess-noise reduction in thin heterojunction $\mathrm{Al}_{0.6} \mathrm{Ga}_{0.4} \mathrm{As}-\mathrm{GaAs}$ avalanche photodiodes," IEEE J. Quantum Electron., vol. 39, pp. 1287-1296, Oct. 2003.

[22] C. Groves, D. S. Ong, J. P. R. David, and G. J. Rees, Modeling of Avalanche Multiplication and Noise Across a Heterojunction.

[23] M. M. Hayat, Ü. Sakoğlu, O. Kwon, S. Wang, J. C. Campbell, B. E. A. Saleh, and M. C. Teich, "Breakdown probabilities for thin heterostructure avalanche photodiodes," IEEE J. Quantum Electron., vol. 39, pp. 179-185, Jan. 2003

[24] S. Wang, F. Ma, X. Li, G. Karve, X. Zheng, and J. C. Campbell, "Analysis of breakdown probabilities in avalanche photodiodes using a history-dependent analytical model," Appl. Phys. Lett., vol. 82, pp. 1971-1973, Mar. 2003

[25] Y. Okuto and C. R. Crowell, "Inoization coefficients in semiconductors a nonlocalized property," Phys. Rev. B, Condens. Matter, vol. 10, pp. 4284-4296, Nov. 1974.

[26] M. M. Hayat, B. E. A. Saleh, and M. C. Teich, "Effect of dead space on gain and noise of double-carrier-multiplication avalanche photodiodes," IEEE Trans. Electron Devices, vol. 39, pp. 546-552, Mar. 1992.

[27] B. I. Cantor and M. C. Teich, "Dead-time-corrected photocounting distributions for laser radiation," J. Opt. Soc. Amer., vol. 65, pp. 786-791, 1975.

[28] B. E. A. Saleh, Photoelectron Statistics. New York: Springer-Verlag, 1978, pp. 272-279.

[29] P. R. Prucnal and M. C. Teich, "Refractory effects in neural counting processes with exponentially decaying rates," IEEE Trans. Syst., Man, Cybernetics, vol. SMC-13, pp. 1028-1033, 1983.

[30] A. Spinelli, A. Pacelli, and A. L. Lacaita, "Dead space approximation for impact ionization in silicon," Appl. Phys. Lett., vol. 69, pp. 3707-3709, 1996.

[31] D. S. Ong, K. F. Li, S. A. Plimmer, G. J. Rees, J. P. R. David, and P N. Robson, "Full band Monte Carlo modeling of impact ionization, avalanche multiplication, and noise in submicron GaAs $p^{+}-i-n^{+}$ diodes," J. Appl. Phys., vol. 87, pp. 7885-7891, June 2000.

[32] S. A. Plimmer, J. P. R. David, B. Jacob, and G. J. Rees, "Impact ionization probabilities as functions of two-dimensional space and time," $J$. Appl. Phys., vol. 89, pp. 2742-2751, Mar. 2001.

[33] M. C. Teich, L. Matin, and B. I. Cantor, "Refractoriness in the maintained discharge of the cat's retinal ganglion cell," J. Opt. Soc. Amer. vol. 68, pp. 386-402, 1978.

[34] N. Sano, T. Aoki, and A. Yoshii, "Soft and hard ionization thresholds in Si and GaAs," Appl. Phys. Lett., vol. 55, pp. 1418-1420, Oct. 1989

[35] S. A. Plimmer, J. P. R. David, D. S. Ong, and K. R. Li, "A simple mode for avalanche multiplication including deadspace effect," IEEE Trans. Electron Devices, vol. 46, pp. 769-775, Apr. 1999.
[36] M. C. Teich and P. Diament, "Relative refractoriness in visual information processing," Biol. Cybernetics, vol. 38, pp. 187-191, 1980.

[37] C. H. Tan, J. P. R. David, G. J. Rees, and R. C. Tozer, "Treatment of soft threshold in impact ionization," J. Appl. Phys., vol. 90, pp. 2538-2543, Sep. 2001

[38] C. L. Anderson and C. R. Crowell, "Threshold energies for electron-hole pair production by impact ionization in semiconductors," Phys. Rev. B, Condens. Matter, vol. 5, pp. 2267-2272, Mar. 1972.

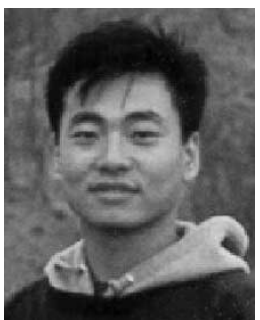

Oh-Hyun Kwon was born in Seoul, Korea, in 1968 $\mathrm{He}$ received the B.S. degree in physics from the University of the Hawaii, Manoa, in 1995, and the M.S. degree in electro-optics from the University of Dayton, Dayton, OH, in 1999. He is currently pursuing the Ph.D. degree in electrical and computer engineering at the University of New Mexico, Albuquerque.

His research interests include modeling and fabrication of optoelectronic devices, with emphasis on APDs and quantum-dot IR detectors.

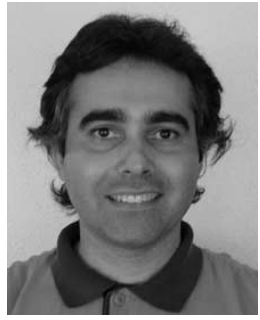

Majeed M. Hayat (S'89-M'92-SM'00) was born in Kuwait in 1963. He received the B.S. degree (summa cum laude) in electrical engineering from the University of the Pacific, Stockton, CA, in 1985, and the M.S. degree in electrical engineering and the $\mathrm{Ph} . \mathrm{D}$. degree in computer engineering from the University of Wisconsin-Madison in 1988 and 1992, respectively.

From 1993 to 1996, he was at the University of Wisconsin-Madison as a Research Associate and Co-Principal Investigator of a project on statistical minefield modeling and detection, which was funded by the Office of Naval Research. In 1996, he joined the faculty of the Electro-Optics Graduate Program and the Department of Electrical and Computer Engineering at the University of Dayton, Dayton, $\mathrm{OH}$. He is currently an Associate Professor in the Department of Electrical and Computer Engineering at the University of New Mexico, Albuquerque. His research interests include modeling and design of high-performance photodetectors, optical communication systems, statistical communication theory, communication networks, infrared imaging and sensors, and statistical signal and image processing.

Dr. Hayat is a member of SPIE and the Optical Society of America. He was the recipient of a 1998 National Science Foundation Early Faculty Career Award.

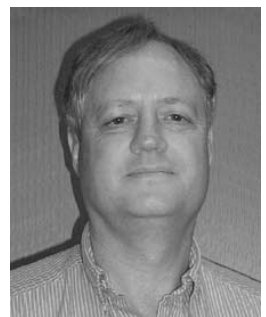

Joe C. Campbell (S'73-M'74-SM'88-F'90) received the B.S. degree in physics from the University of Texas at Austin in 1969, and the M.S. and Ph.D. degrees in physics from the University of Illinois at Urbana-Champaign in 1971 and 1973, respectively.

From 1974 to 1976, he was with Texas Instruments, where he worked on integrated optics. In 1976, he joined the staff of AT\&T Bell Laboratories, Holmdel, NJ. In the Crawford Hill Laboratory, he worked on a variety of optoelectronic devices including semiconductor lasers, optical modulators, waveguide switches, photonic integrated circuits, and photodetectors with emphasis on high-speed APDs for high-bit-rate lightwave systems. In January 1989, he joined the faculty of the University of Texas at Austin as Professor of Electrical and Computer Engineering and Cockrell Family Regents Chair in Engineering. Currently, he is actively involved in Si-based optoelectronics, high-speed APDs, GaN ultraviolet photodetectors, and quantum-dot IR imaging. He has coauthored six book chapters, more than 260 journal publications, and 160 conference presentations.

Dr. Campbell is a Fellow of the Optical Society of America. In 1985, he was recognized by AT\&T as Distinguished Member of Technical Staff. In 2002, he was elected into the National Academy of Engineering. 


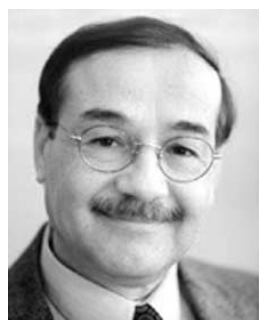

Bahaa E. A. Saleh (M'73-SM'86-F'91) received the B.S. degree from Cairo University, Cairo, Egypt, in 1966, and the Ph.D. degree from Johns Hopkins University, Baltimore, MD, in 1971, both in electrical engineering.

Since 1994, he has been Professor and Chairman of the Department of Electrical and Computer Engineering at Boston University, Boston, MA. $\mathrm{He}$ is Deputy Director of the NSF Center for Subsurface Sensing and Imaging Systems, an NSF Engineering Research Center. He is also Co-Director of the Quantum Imaging Laboratory and a member of the Boston University Photonics Center. He held faculty and research positions at the University of Santa Catarina in Brazil, Kuwait University, Max Planck Institute in Germany, the University of California-Berkeley, the European Molecular Biology Laboratory, Columbia University, and the University of Wisconsin-Madison, where he was a faculty member from 1977 to 1994, and served as Chairman of the Department of Electrical and Computer Engineering from 1990 to 1994. His research contributions cover a broad spectrum of topics in optics and photonics including statistical and quantum optics, optical communication and signal processing, nonlinear optics, photodetectors, digital image processing, and vision. He is the author of two books, Photoelectron Statistics (New York: Springer-Verlag, 1978) and Fundamentals of Photonics (New York: Wiley-InterScience, 1991, with M. C. Teich), chapters in seven books, and more than 180 papers in technical journals. He served as Editor-in-Chief of the Journal of the Optical Society of America A from 1991 to 1997, and is presently the Chairman of the Board of Editors of the Optical Society of America.

Dr. Saleh is a Fellow of the Optical Society of America and the Guggenheim Foundation. He is the recipient of the 1999 Optical Society of America Esther Hoffman Beller Award for outstanding contributions to optical science and engineering education. He is also a member of Phi Beta Kappa, Sigma Xi, and Tau Beta Pi.

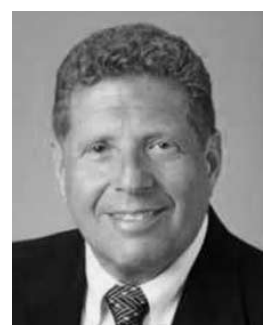

Malvin C. Teich (S'62-M'66-SM'72-F'89) received the B.S. degree in physics from the Massachusetts Institute of Technology (MIT), Cambridge, the M.S. degree in electrical engineering from Stanford University, Stanford, CA, and the Ph.D. degree from Cornell University, Ithaca, NY.

His first professional association was with MIT Lincoln Laboratory, Cambridge, in 1966. He joined the faculty at Columbia University in 1967, where he served as a member of the Department of Electrical Engineering (as Chairman from 1978 to 1980), the Department of Applied Physics, and the Columbia Radiation Laboratory. During his tenure at Columbia, he carried out extensive research in the areas of noise in APDs and fiber-optic amplifiers, photon statistics and point processes, and the generation of squeezed light. In 1996, he was appointed Professor Emeritus of Engineering Science and Applied Physics at Columbia University. Since 1995, he has been teaching and pursuing his research interests at Boston University, Boston, MA, as a faculty member with joint appointments in the Departments of Electrical and Computer Engineering, Physics, and Biomedical Engineering. He is a member of the Quantum Imaging Laboratory, the Photonics Center, and the Center for Adaptive Systems. He also serves as a Consultant to government and private industry. He is most widely known for his work in photonics and quantum optics. His current efforts in photonics are associated with the reduction of noise in APDs and fiber-optic amplifiers; his efforts in quantum optics are directed toward developing imaging systems that make use of the correlation properties of entangled photon pairs generated in nonlinear optical parametric downconversion. He has authored or coauthored approximately 300 technical publications and holds two patents. He is the coauthor of Fundamentals of Photonics (New York: Wiley, 1991). He served as a member of the Editorial Advisory Panel for the journal Optics Letters from 1977 to 1979, as a member of the Editorial Board of the Journal of Visual Communication and Image Representation from 1989 to 1992, and as Deputy Editor of Quantum Optics from 1988 to 1994. He is currently a member of the Editorial Board of the journal Jemná Mechanika a Optika.

Dr. Teich is a Fellow of the Optical Society of America, the American Physical Society, the American Association for the Advancement of Science, and the Acoustical Society of America. He is a member of Sigma Xi and Tau Beta $\mathrm{Pi}$. He is also a member of the Scientific Board of the Czech Academy of Sciences' Institute of Physics. In 1969, he received the IEEE Browder J. Thompson Memorial Prize for his paper "Infrared Heterodyne Detection." He was awarded a Guggenheim Fellowship in 1973. In 1992, he was honored with the Memorial Gold Medal of Palacký University in the Czech Republic and in 1997, he was the recipient of the IEEE Morris E. Leeds Award. He is currently a member of the Advisory Board of the IEEE Press Series Emerging Technologies in Biomedical Engineering. 